\title{
Jet Structure in the Afterglow Phase for Gamma-ray Bursts with a Precessing Jet
}

\author{
Bao-Quan Huang, ${ }^{1}$ Da-Bin Lin, ${ }^{1 \star}$ Tong Liu, ${ }^{2}$ Jia Ren,${ }^{1}$ Xiang-Gao Wang, ${ }^{1}$ \\ Hong-Bang Liu, ${ }^{1}$ En-Wei Liang ${ }^{1}$
}

${ }^{1}$ Laboratory for Relativistic Astrophysics, Department of Physics, Guangxi University, Nanning 530004, China

${ }^{2}$ Department of Astronomy, Xiamen University, Xiamen, Fujian 361005, China

Accepted XXX. Received YYY; in original form ZZZ

\begin{abstract}
The structured jet is involved to explain the afterglows and even the prompt emission of GRB 170817A. In this paper, we stress that for a precessing jet, the jet structure in the prompt emission phase and that in the afterglow phase may be different. The jet structure in the afterglow phase can be non-uniform even if a narrow-uniform jet is presented in the prompt emission phase. We estimate the jet structure in the afterglow phase under the situation that a narrow-uniform-precessing jet is launched from the central engine of gamma-ray burst. With different precession angles, it is found that the structured jet can be roughly described as follows: a narrow uniform core with power-law wings and sharp cut-off edges, a Gaussian profile, a ring shape, or other complex profile in energy per solid angle. Correspondingly, the afterglows for our obtained structured jets are also estimated. We find that the estimates of the intrinsic kinetic energy, the electron index, and the jet opening angle based on the afterglows formed in a precessing system may be incorrect. Our obtained structured jet is likely to be revealed by future observations for a fraction of gravitational wave detected merging compact binary systems (e.g., black hole-neutron star mergers).
\end{abstract}

Key words: gamma-ray burst: general - gravitational waves - gamma-ray burst: individual (GRB 170817A)

\section{INTRODUCTION}

Gamma-ray bursts (GRBs) are the most powerful electromagnetic explosions in the universe. They are widely argued to originate from the compact binaries mergers or core collapse of massive stars. On August 17, 2017 at 12:41:04 UTC, the advanced Laser Interferometer Gravitationalwave Observatory and the Advanced Virgo gravitationalwave detectors made their first detection of a gravitational wave event (GW 170817) from the merger of a binary neutron star system (Abbott et al. 2017a; Abbott et al. 2017b; Abbott et al. 2017c; Abbott et al. 2017d). About 2 s post-merger, the Fermi (Goldstein et al. 2017) and INTEGRAL satellites (Savchenko et al. 2016) observed a short burst (GRB 170817A) from a location coincident with GW 170817. A series of observation campaigns following this discovery have led to the detection of a bright optical counterpart, AT2017gfo (Arcavi et al. 2017;

* E-mail: lindabin@gxu.edu.cn
Coulter et al. 2017; Kasen et al. 2017; Kasliwal et al. 2017; Pian et al. 2017; Smartt et al. 2017; Soares-Santos et al. 2017; Valenti et al. 2017; Hu et al. 2017; Tanvir et al. 2017; Lipunov et al. 2017) associated with the kilonova powered by the radioactive decay of heavy elements formed in the binary neutron star merger (Li \& Paczyński 1998; Metzger \& Berger 2012; Berger, Fong, \& Chornock 2013; Fernández \& Metzger 2016; Song, Liu, \& Li 2018; Liu, Gu, \& Zhang 2017). The joint GW-GRB detection has provided the first compelling observational evidence on the relation of short GRBs and the binary neutron star mergers.

The physical origin of GRB 170817A emission is still under debate. The prompt $\gamma$-rays of GRB $170817 \mathrm{~A}$ is argued to originate from the photosphere of jets (Meng et al. 2018), the internal shocks (Murguia-Berthier et al. 2017; Fraija et al. 2017), the internal-collision-induced magnetic reconnection and turbulence (Meng et al. 2018; Zhang \& Yan 2011), or the external-reverse shock (Fraija et al. 2017). Meanwhile, a diverse sets of jet structure are involved, e.g., an off-axis top-hat jet (Lin et al. 2018), 
a mildly relativistic and isotropic fireball, and a structured jet (e.g., D'Avanzo et al. 2018; Lamb \& Kobayashi 2018; Lazzati et al. 2018; Lyman et al. 2018; Margutti et al. 2018; Meng et al. 2018; Resmi et al. 2018; Troja et al. 2018). These models should explain both the radiation spectrum and the lag $\left(t_{\text {lag }} \sim 2 \mathrm{~s}\right)$ of GRB prompt emission relative to GW 170817. An off-axis top-hat jet launched immediately after the merger can naturally produce the relation of $t_{\text {lag }} \sim T_{90}$ with $T_{90}$ being the duration of prompt $\gamma$-ray emission (Lin et al. 2018). However, $t_{\text {lag }}$ is mainly caused by the delay of the merger and jet launching under the framework of photosphere model (Zhang, et al. 2018; Meng et al. 2018) or is corresponding to the shock breakout from a cocoon (Gottlieb et al. 2018). The prompt emission of GRB 170817A may be the scattered emission of a short GRB by a cocoon and the values of $t_{1 \mathrm{ag}}$ and $T_{90}$ are reproduced with typical short GRB parameters (Kisaka et al. 2018). Apart from the prompt emission, the broad-band afterglows of GRB 170817A is peculiar but such kind of afterglows was predicted before GW 170817/GRB 170817A (Lamb \& Kobayashi 2017). Nonetheless, the origin of the outflow structure is still open to debate. The afterglows were first detected at $\sim 9$ days by Chandra in the X-rays (Haggard et al. 2017; Evans et al. 2017; Margutti et al. 2018; Troja et al. 2017) and $\sim 16$ days in the radio band (Hallinan et al. 2017) after GW 170817. Its spectrum can be described as a single power-law for radio-optical-X-ray observations (Lyman et al. 2018), which is consistent with the emission from a relativistic external-forward shock. However, the afterglows continued to rise in flux until ¿ 115 days after GW 170817 (e.g., Lyman et al. 2018; Margutti et al. 2018; Mooley et al. 2018; Ruan et al. 2018; Troja et al. 2017). The continued brightening is anomalous for canonical GRBs and in favor of the structured jet scenario. Recently, Troja et al. (2018) found that a Gaussian profile jet with an off-axis observer is successful in capturing the observed features of GRB 170817A for a year-long afterglow monitoring (see also Lamb, et al. 2019; van Eerten, et al. 2018). Thus, the structured jets are involved to explain the afterglows and even the prompt $\gamma$-rays of GRB 170817A (see the discussion in the beginning of this paragraph). In this paper, however, we would like to point out that the jet structure in the prompt emission phase may be very different from that in the afterglow emission phase, especially in GRBs with a precessing jet. For GRBs with a narrow-uniform-precessing jet, a structured jet is difficult to form in the prompt emission phase due to the low frequency of mergers between jet shells. In the afterglow phase, the early launched jet shells are decelerated during its propagation into the circum-burst medium. Thus, the later launched jet shells can catch up and collide with the early launched ones in the early phase of afterglow. In the situation with a precessing jet, a structured jet can be easily formed in the afterglow phase.

We study the jet structure in the afterglow phase under the situation that a precessing jet is launched from the central engine of GRBs. The paper is organized as follows. The procedure to calculate the jet structure and the obtained structured jet are shown in Sections 2 and 3, respectively. The conclusions and discussion are presented in Section 4 .

\section{PROCEDURE TO CALCULATE THE JET STRUCTURE}

Jet precession has been previously discussed as a phenomenon relevant for GRBs (e.g., Blackman, Yi, \& Field 1996; Portegies Zwart, Lee, \& Lee 1999; Reynoso, Romero, \& Sampayo 2006; Lei et al. 2007; Foucart et al. 2011; Stone, Loeb, \& Berger 2013; Liu, Gu, \& Zhang 2017; Liska et al. 2018). The large amplitude precession requires large amplitude misalignment of the post-merger black hole $(\mathrm{BH})$ and its accretion disk. In $\mathrm{BH}-$ neutron star (NS) mergers, the $\mathrm{BH}$ may possess a larger natal reservoir of spin angular momentum, allowing for greater misalignment between the post-merger $\mathrm{BH}$ and the disk formed from NS debris. Then, Stone, Loeb, \& Berger (2013) stated that the precession can carry significant observational consequences for BH-NS mergers. However, the situation for NS-NS mergers is different except a millisecond spin NS is involved in the binaries (Stone, Loeb, \& Berger 2013). GW 170817 is the first detected GW signal from a NSNS merger (but see Hinderer et al. 2018 for BH-NS merger origin of GW 170817). Then, the precession may be likely too small to carry significant observational consequences in GRB 170817A. The detail mechanisms being response to the jet precession can refer to Stone, Loeb, \& Berger (2013). If a spinning black hole is surrounded by a tilted accretion disk, a precessing jet may be launched from the central engine of GRBs. The schematic picture is shown in Figure 1, where the central engine of GRBs locates at the origin of coordinate $(r=0)$. The yellow region represents the narrow-uniform-precessing jet with opening angle $\theta_{\text {open }}$, which rotates around $z$-axis with a precession angle $\theta_{\text {pre }}$. The spherical coordinate $(r, \theta, \phi)$ with $\theta=0$ being along the direction of $z$-axis is used in this paper, $\left(\theta_{\text {obs }}, \phi_{\text {obs }}\right)$ is adopted to describe the direction of the observer, and $\left(\theta_{\text {pre }}, \phi_{\text {pre }}\right)$ represents the direction of the jet axis at time $t$. Due to the jet precession, the total output energy of jet would be distributed into a large solid angle. This behavior depends on the precession period $\tau$ and the evolution behavior of the jet power $P(t)$.

In this work, we compute the final jet structure, i.e., the energy distribution $\varepsilon(\theta, \varphi)$, for a narrow-uniform-precessing jet. The procedures to obtain the jet structure are shown as follows. We divide the time $t$ into a series of time intervals $[0, \delta t],[\delta t, 2 \delta t], \ldots,[(k-1) \delta t, k \delta t], \ldots,\left[t_{\mathrm{end}}-\delta t, t_{\mathrm{end}}\right]$ with $t_{\text {end }}=K \delta t$ being the duration of jet activities. For each time interval, the total output energy of the jet can be estimated with $P(t=k \delta t-\delta t / 2) \delta t$, which will be redistributed to $n$ infinitesimal-ejecta-cells (IECs). Here, the solid angle of an IEC is set to zero and the energy of an IEC is $P(t=k \delta t-\delta t / 2) \delta t / n$, which is different for different time interval since $P(t)$ is a time-dependent function. In the $k$ th time interval, we launch $N=2 n /\left(1-\cos \theta_{\text {open }}\right)$ IECs with randomly selected direction $\left(\theta_{\text {cell }}, \phi_{\text {cell }}\right)$, where the value of $\cos \theta_{\text {cell }}$ and $\phi_{\text {cell }}$ are randomly took in the range of $[-1,1]$ and $\left[0^{\circ}, 360^{\circ}\right]$, respectively. For these $N(\gg 1)$ cells, only $\sim n$ IECs fall into the solid angle of the jet shell at time $t=(k-1 / 2) \delta t$ and are used to represent the energy output of the jet in the $k$ th time interval. We select out these $\sim n$ IECs by using the following relation:

$\cos \theta_{\text {open }} \leqslant \sin \theta_{\text {cell }} \cos \phi_{\text {cell }} \sin \theta_{\text {pre }} \cos \phi_{\text {pre }}+$
$\quad \sin \theta_{\text {cell }} \sin \phi_{\text {cell }} \sin \theta_{\text {pre }} \sin \phi_{\text {pre }}+\cos \theta_{\text {cell }} \cos \theta_{\text {pre }}$, 


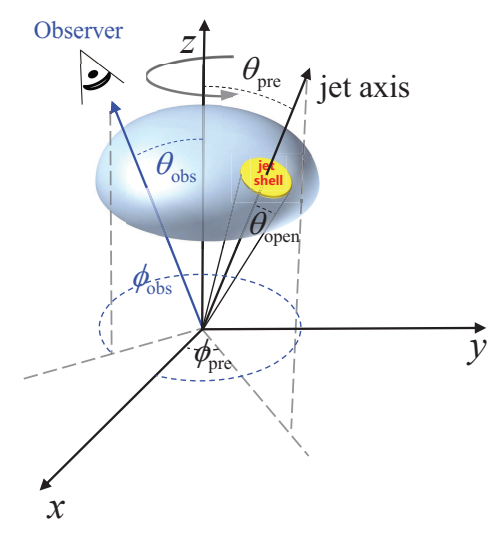

Figure 1. Schematic picture of a precessing jet in GRBs.

where $\left(\theta_{\text {pre }}, \phi_{\text {pre }}\right)$ describes the direction of the jet axis at time $t=(k-1 / 2) \delta t$. If $N \gg 1$ is satisfied, the total energy of our selected IECs at time $t=(k-1 / 2) \delta t$ would be pretty close to $P(t) \delta t$, which is the total output energy in the $k$ th time interval. By changing $k$ from 1 to $K$, we can select out a series of IECs with different energy and different direction. We divide the solid angle of $\theta \in\left[\max \left\{\theta_{\text {pre }}-\theta_{\text {open }}, 0\right\}, \min \left\{\theta_{\text {pre }}+\theta_{\text {open }}, \pi / 2\right\}\right]$ and $\phi \in[0,2 \pi]$ into $500 \times 200$ grids. Our selected IECs would fall into different grids. The total energy of a grid is obtained by summing up the energy of these IECs which fall into the corresponding grid. By dividing the grid's total energy to the grid's solid angle, one can obtain the energy density $\varepsilon$ per solid angle for grids.

\section{RESULTS}

We first study the jet structure with $P(t)=$ constant, $t_{\text {end }}=\tau$, and different $\theta_{\text {pre }}$. The obtained distribution of energy density $\varepsilon$ per solid angle is shown in Figure 2, where $\varepsilon_{\max }$ is the maximum energy density and $\theta_{\text {pre }}=2.5^{\circ}$ (left panel), $5^{\circ}$ (middle panel), and $10^{\circ}$ (right panel) are adopted, respectively. In this figure, the upper sub-figures in each panel present the dependence of $\varepsilon$ on $x=\sin \theta \cos \phi$ and $y=\sin \theta \sin \phi$. For a given $\theta$ in these sub-figures, i.e., $x^{2}+y^{2}=$ constant, the value of $\varepsilon$ is the same for different $\phi$. This can be easily found in the middle sub-figures of each panel, which show the dependence of $\varepsilon$ on $\theta$ and $\phi$. In the situation of $\theta_{\text {open }}>\theta_{\text {pre }}$ (left panel), one can find that $\varepsilon$ remains constant in the region with $\theta<\theta_{\text {open }}-\theta_{\text {pre }}$ and follows a power-law decay in the region of $\theta>\theta_{\text {open }}-\theta_{\text {pre }}$. Since the $\varepsilon$ does not evolve in $\phi$-direction, we adopt the following function to fit the energy distribution:

$$
\frac{\varepsilon(\theta)}{\varepsilon_{\max }}=\left\{\begin{array}{cc}
1, & \theta \leq \theta_{\mathrm{c}} \\
\left(\theta / \theta_{\mathrm{c}}\right)^{-k}, & \theta_{\mathrm{c}}<\theta<\theta_{\mathrm{m}}
\end{array}\right.
$$

where $\theta_{\mathrm{c}}$ and $\theta_{\mathrm{m}}$ are constants. The result is shown with red solid line in Figure 2, and can be read as $\theta_{\mathrm{c}}=2.5^{\circ}, \theta_{\mathrm{m}}=5^{\circ}$, and $k=1.05$. Since the jets in Figure 2 are calculated with a narrow-uniform-precessing jet, the Lorentz factor $\Gamma(\theta, \phi)$ of our obtained jet should be the same for different $(\theta, \phi)$, i.e., $\Gamma(\theta, \phi)=$ constant. This is different from the structured jet adopted in Xiao et al. (2017) (see also, e.g., Dai \& Gou 2001; Zhang \& Mészáros 2002; Rossi, Lazzati, \& Rees 2002;
Kumar \& Granot 2003). For $\theta>\theta_{\mathrm{m}}$ in the left panel, the $\varepsilon$ suffers from a sharp cut-off decay. Then, the Gaussian function, i.e.,

$$
\varepsilon(\theta) \propto \exp \left[-\left(\theta-\theta_{0}\right)^{2} / 2 \theta_{\mathrm{g}}^{2}\right]
$$

is adopted to fit this part and the result (green dashed line) can be read as $\theta_{0}=4.8^{\circ}$ and $\theta_{\mathrm{g}}=1.6^{\circ}$. In summary, the structured jet in the left panel can be described as

$$
\left\{\begin{array}{cc}
\frac{\varepsilon(\theta)}{\varepsilon_{\max }}= & \theta \leq \theta_{\mathrm{c}}, \\
1, & \theta_{\mathrm{c}}<\theta \leq \theta_{\mathrm{m}}, \\
\left(\frac{\theta}{\theta_{\mathrm{c}}}\right)^{-k}, & \theta_{\mathrm{m}}<\theta<\theta_{\text {open }}+\theta_{\text {jet }} .
\end{array}\right.
$$

According to Figure 2, it can be easily found that a powerlaw function could not describe the energy distribution for the other two situations. In addition, the cut-off behavior is obviously in these two situations. Then, we adopt the Gaussian function to fit the energy distribution. The results are shown with green dashed lines, i.e., $\left(\theta_{0}, \theta_{\mathrm{g}}\right)=\left(1.6^{\circ}, 5.0^{\circ}\right)$ for $\theta_{\text {open }}=\theta_{\text {pre }}$ and $\left(\theta_{0}, \theta_{\mathrm{g}}\right)=\left(9.7^{\circ}, 3.1^{\circ}\right)$ for $\theta_{\text {open }}<\theta_{\text {pre }}$. One can find that the Gaussian function can better describe the jet structure for the situation of $\theta_{\text {pre }}=5^{\circ}$. However, it fails to describe the jet structure for the situation with $\theta_{\text {pre }}=10^{\circ}$. The energy distribution in the situation of $\theta_{\text {open }}<\theta_{\text {pre }}$ is a ring-shaped jet, but not a uniform ring-shaped jet (Granot 2005; Zou \& Dai 2006;0 Xu, Huang, \& Kong 2008; Xu \& Huang 2010).

We estimate the X-ray $(0.3-10 \mathrm{keV})$ emission of the external-forward shock for our obtained structured jet in Figure 3. The obtained X-ray light curves are shown in Figure 3 with solid lines, where the same total energy of the structured jet is adopted. That is to say, the values of $\varepsilon_{\max }=4.17 \times 10^{53} \mathrm{erg}, 1.91 \times 10^{53} \mathrm{erg}$, and $7.0 \times 10^{52} \mathrm{erg}$ are adopted for the situations with $\theta_{\text {pre }}=2.5^{\circ}, 5^{\circ}$, and $10^{\circ}$, respectively. The dynamics of external-forward shock can refer to Huang, Dai, \& Lu (1999), and the value of other parameters to calculate the afterglow emission are the electron equipartition parameter $\epsilon_{e}=0.1$, the magnetic equipartition parameter $\epsilon_{B}=0.01$, the electron power-law index $p=2.5$, the interstellar medium density $n=0.01$, the Lorentz factor $\Gamma(\theta, \phi)=200$, and the luminosity distance $D_{\mathrm{L}}=40 \mathrm{Mpc}$. The afterglows are obtained by summing the resulting emission of $500 \times 200$ grids (see Section 2 ) with different inclination to the light-of-sight. The viewing angle $\left(\theta_{\mathrm{obs}}, \phi_{\mathrm{obs}}\right)$ with $\theta_{\text {obs }}=0^{\circ}, 5^{\circ}, 10^{\circ}, 20^{\circ}, 40^{\circ}$, and $60^{\circ}$ are shown with red, green, blue, cyan, magenta, and yellow lines, respectively. Here, the value of $\phi_{\mathrm{obs}}$ does not affect the profile of light curves and $\phi_{\mathrm{obs}}=0^{\circ}$ is adopted. For comparison, the $\mathrm{X}$-ray afterglows from the situation with $\theta_{\text {pre }}=0$ is also shown with "o" in Figure 3 and the afterglows with the same viewing angle are plotted with the same color. According to Figure 3 , the light curve of afterglows can be very different for different $\theta_{\text {pre }}$, especially for the situation with $\theta_{\text {pre }} \gtrsim \theta_{\text {open }}$. In addition, the flux in the normal decay phase of afterglows decreases with increasing the value of $\theta_{\text {pre }}$ for situations with $\theta_{\text {pre }}<\theta_{\text {open }}$ and $\theta_{\text {obs }}=0^{\circ}$. This behavior may affect the estimated kinetic energy of the external shock.

In the following part, we study the jet structure for a precessing jet with an evolving $P(t)$. The obtained jet structure would depend on the precession period $\tau$ and the evolution behavior of the jet power $P(t)$. For a jet powered via 


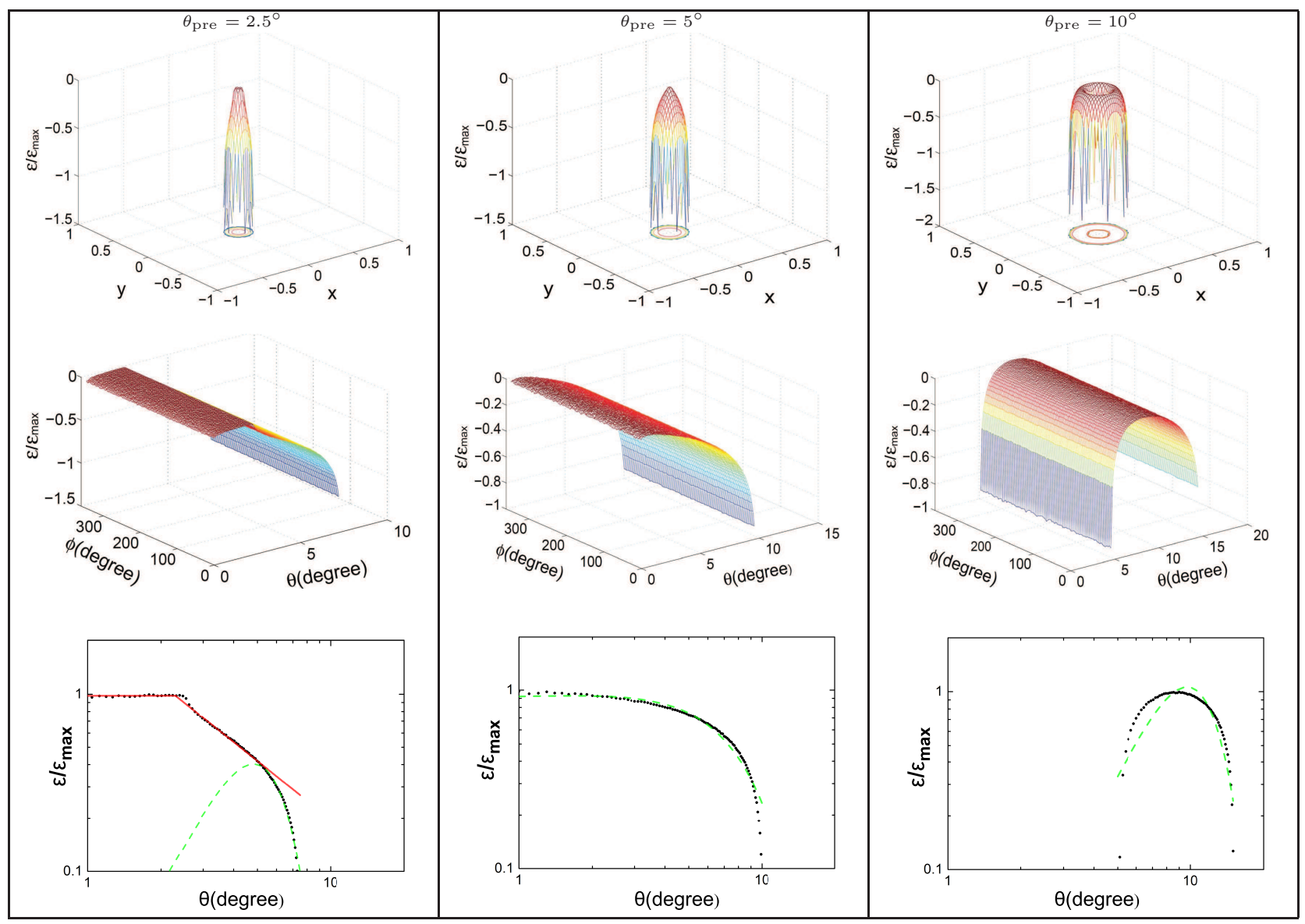

Figure 2. Distribution of energy density $\varepsilon$ for GRBs with a precessing jet. From left to right panel, the value of $\theta_{\text {pre }}=2.5^{\circ}, 5^{\circ}$, and $10^{\circ}$ are adopted, respectively. The dependence of $\varepsilon$ on $(x, y),(\theta, \phi)$, and $\theta$ are shown in the upper, middle, and lower sub-figures in each panel, respectively.
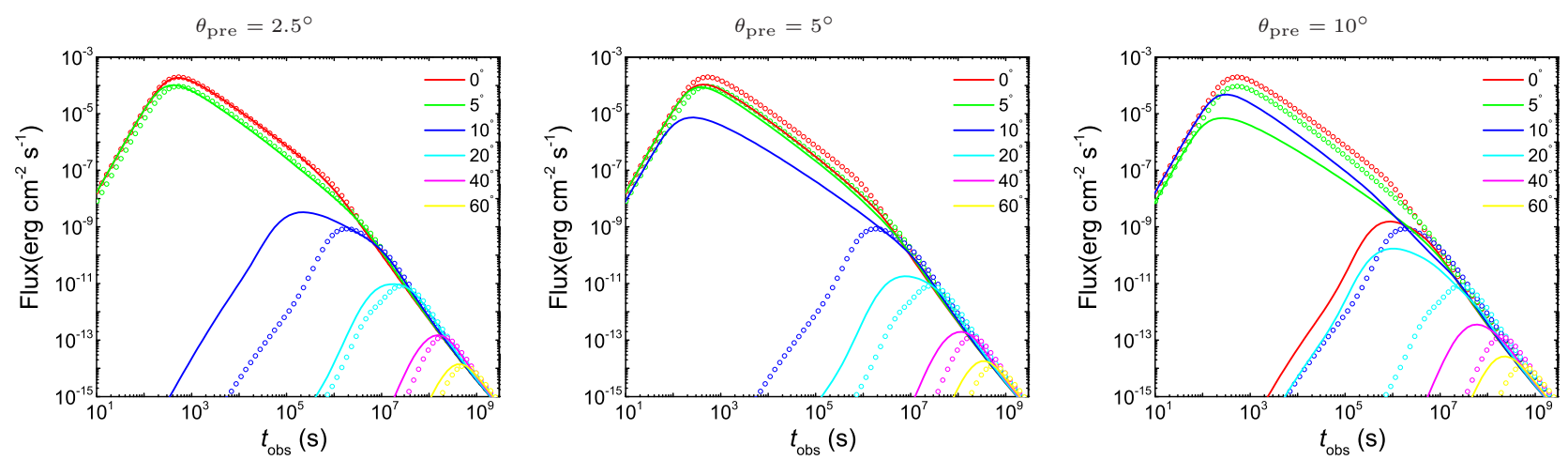

Figure 3. The X-ray afterglows for GRBs with a precessing jet and different $\theta_{\text {pre, }}$, where the viewing angle $\theta_{\mathrm{v}}=0^{\circ}$ (red solid line), $5^{\circ}$ (green solid line), $10^{\circ}$ (blue solid line), $20^{\circ}$ (cyan solid line), $40^{\circ}$ (magenta solid line), and $60^{\circ}$ (yellow solid line) are adopted. The "o" represents the X-ray afterglows from the situation with $\theta_{\text {pre }}=0^{\circ}$ and the afterglows with the same viewing angle are plotted with the same color.

Blandford \& Znajek (1977) mechanism, the jet power depends on the black hole (BH) mass $M$, the $\mathrm{BH}$ spin $a$, and the magnetic field $B$ accumulated near the BH horizon. Since the magnetic field on the $\mathrm{BH}$ is supported by the accretion disk $\dot{M}$, it is reasonable to assume $B^{2} \propto \dot{M}$ (Wu, Hou, \& Lei 2013). If $M$ and $a$ remain constant, the evolution of jet power can be described as (e.g., Wu, Hou, \& Lei 2013; Yu et al.
2015)

$$
P_{\text {jet }}(t) \sim P_{0}\left[\frac{1}{2}\left(\frac{t}{t_{\mathrm{r}}}\right)^{-a_{\mathrm{r}} s}+\frac{1}{2}\left(\frac{t}{t_{\mathrm{d}}}\right)^{-a_{\mathrm{d}} s}\right]^{-1 / s}
$$

with $P_{0}=10^{52} \mathrm{erg} / \mathrm{s}, a_{\mathrm{r}}=1 / 2, a_{\mathrm{d}}=-5 / 3, s=6, t_{\mathrm{r}}=$ $0.1 \tau$, and $t_{\text {end }}=10 \tau$. We adopt the following two case to discuss the dependence of the jet structure on $\tau$ and $P(t)$ : 


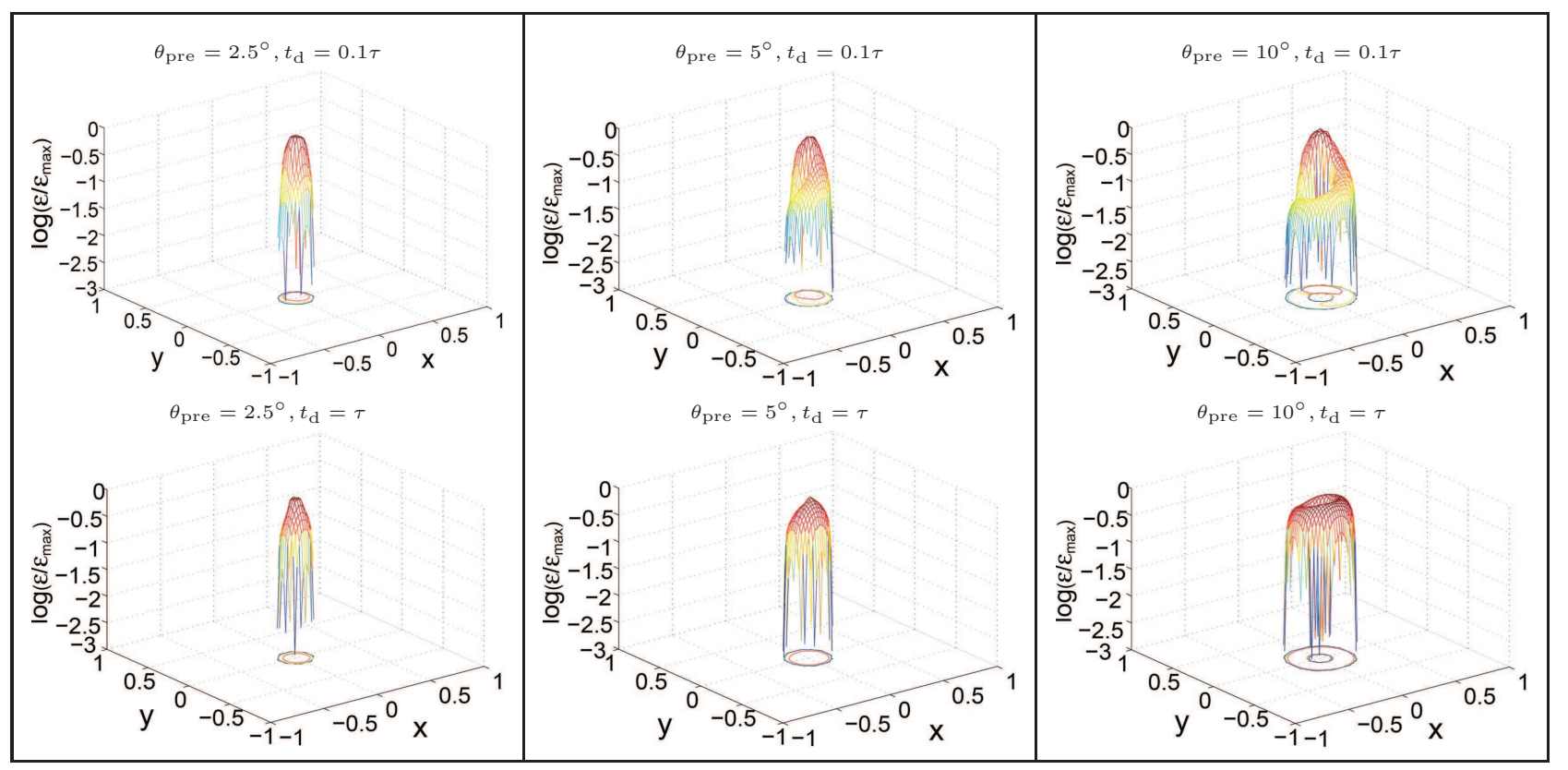

Figure 4. Dependence of $\varepsilon$ on $(x, y)$ for a precessing jet with an evolving $P(t)$.
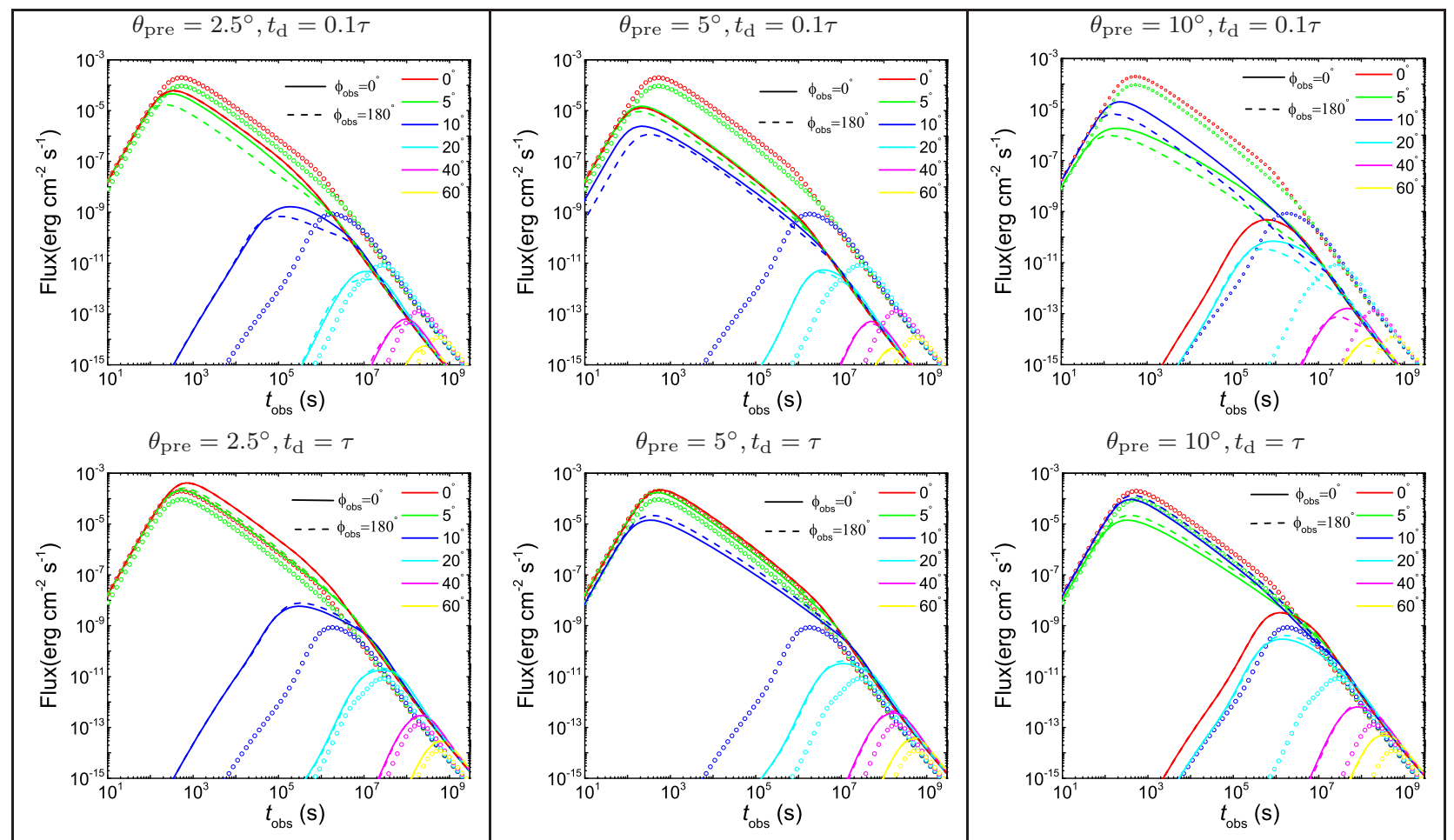

Figure 5. The X-ray afterglows for an evolving jet with $t_{\mathrm{d}}=0.1 \tau$ (upper sub-figures) and $t_{d}=\tau$ (lower sub-figures),respectively. The solid and dashed lines represent the results for an observer with viewing angle of $\left(\theta_{\text {obs }}, \phi_{\text {obs }}\right)=\left(\theta_{\text {obs }}, 0^{\circ}\right)$ and $\left(\theta_{\text {obs }}, 180^{\circ}\right)$, respectively. Here, $\theta_{\text {obs }}=0^{\circ}, 5^{\circ}, 10^{\circ}, 20^{\circ}, 40^{\circ}, 60^{\circ}$ are studied in each sub-figure and the meaning of "o" symbol is the same as that in Figure 3.

(I) $t_{\mathrm{d}}=0.1 \tau$, (II) $t_{\mathrm{d}}=\tau$. The obtained energy distribution is shown in Figure 4 , where $\theta_{\text {pre }}=2.5^{\circ}, 5^{\circ}$, and $10^{\circ}$ are adopted in the left, middle, and right panels, respectively. One can find that the jet structure is very complex in these situations. Moreover, the structured jet becomes uniform in $\phi$-direction if the evolution timescale of $P(t)$ is at around or larger than the precession period $\tau$. The X-ray afterglows are also calculated and shown in Figure 5 , where $\theta_{\text {obs }}=0^{\circ}$ (red lines), $5^{\circ}$ (green lines), $10^{\circ}$ (blue lines), $20^{\circ}$ (cyan lines), $40^{\circ}$ (magenta lines), and $60^{\circ}$ (yellow lines) with $\phi_{\text {obs }}=0^{\circ}$ (solid lines) or $180^{\circ}$ (dashed lines) are adopted. The situation with $\theta_{\mathrm{obs}}=0^{\circ}$ and $\phi_{\mathrm{obs}}=0^{\circ}$ is the same as that with $\theta_{\mathrm{obs}}=0^{\circ}$ and $\phi_{\text {obs }}=180^{\circ}$. Then, we only plot the solid line for the situation with $\theta_{\text {obs }}=0^{\circ}$. Here, the "o" is the same as Figure 
3. One can find that the afterglows with $\theta_{\text {pre }} \neq 0^{\circ}$ and those with $\theta_{\text {pre }}=0^{\circ}$ are very different. Moreover, the observer with different azimuthal angle $\phi_{\text {obs }}$ may detect different light curves of afterglows.

\section{CONCLUSIONS AND DISCUSSIONS}

Most structured jet models used to explain the GRB 170817 afterglow can not and should not assume that the structure is the same as that when the GRB is emitted. In this work, we point out that the jet structure in the prompt emission phase can be very different from that in the afterglow phase for GRBs with a precessing jet. For GRBs with a narrow-uniform-precessing jet, a structured jet is difficult to form in the prompt emission phase due to the low frequency of mergers between jet shells. However, the structured jet can be easily formed in the early phase of afterglow. We estimate the jet structure in the afterglow phase under the situation that a narrow-uniform-precessing jet is launched from the central engine of a GRB. With different precession angle, the obtained structured jet can be roughly described as a narrow uniform core with power-law wings and sharp cut-off edges, a Gaussian profile, or a ring shape. For a precessing jet with an evolving jet power $P(t)$, the obtained jet structure is very complex and depends on both the precession period $\tau$ and the evolution behavior of $P(t)$. In addition, the structured jet may be not axisymmetric. The structured jet formed due to the precession of jet is likely to be revealed by future observations for a fraction of GW detected merging compact binary systems, e.g., BH-NS mergers (Stone, Loeb, \& Berger 2013).

We also calculate the X-ray emission of the externalforward shock for our obtained structured jet. Our results show that the X-ray flux decreases with increasing the precession angle. This can be found in the left and middle subfigures of Figure 3 with $\theta_{\text {obs }} \sim 0^{\circ}$. We estimate the kinetic energy of the jet based on the X-ray afterglows for situations with different $\theta_{\text {pre }}\left(<\theta_{\text {open }}\right)$ and $\theta_{\text {obs }}=0^{\circ}$. The result is shown in Figure 6, where the left panel shows our synthetic light curves of afterglow emission at $10 \mathrm{keV}$ and the right panel plots the relation of $E_{\mathrm{k}}$ and $\theta_{\mathrm{pre}}$. From this figure, one can find that the kinetic energy decreases with $\theta_{\text {pre }}$. Then, the larger value of $\theta_{\text {pre }}$ is, the higher radiation efficiency of the GRB prompt emission would be found. This may explain the exorbitant higher radiation efficiency of prompt emission found in some GRBs (e.g., Granot, Königl, \& Piran 2006; Ioka et al. 2006; Nousek et al. 2006; Zhang et al. 2007). The precession of a jet would also lead to incorrect estimates of the electron index. In the left panel of Figure 6, the slope of the afterglow flux in the normal decay phase becomes steep with the increase of the precession angle. Then, the electron index estimated based on the slope of the normal decay phase (Zhang et al. 2006) would become higher with the increase of the precession angle, even though the intrinsic value of the electron index remains constant. In other words, the spectral index and the decay slope of the normal decay phase may deviate from the closure relations of the external-forward shock model (e.g., Zhang \& Mészáros 2004; Zhang et al. 2006) for GRBs with higher precession angle. In addition, the time of jet-break caused by the edge effect is gradually deferred with the increase of the preces- sion angle. The jet break even becomes unclear in the situation with high precession angle. This behavior may help to understand the lack of expected jet breaks in Swift X-ray afterglows (e.g., Racusin, et al. 2009; Wang, et al. 2018). The incorrect estimates about the electron index and the deferred jet break time may affect the estimated GRB total output energy.

\section{ACKNOWLEDGMENTS}

We thank the anonymous referee of this work for beneficial suggestions that improved the paper. This work is supported by the National Natural Science Foundation of China (grant Nos. 11773007, 11533003, 11673006, 11822304, U1731239), the Guangxi Science Foundation (grant Nos. 2018GXNSFFA281010, 2016GXNSFDA380027, 2017AD22006, 2016GXNSFFA380006, 2018GXNSFGA281005), the Innovation Team and Outstanding Scholar Program in Guangxi Colleges, and the OneHundred-Talents Program of Guangxi colleges.

\section{REFERENCES}

Abbott B. P., et al., 2017a, ApJ, 850, L39

Abbott B. P., et al., 2017b, ApJ, 850, L40

Abbott B. P., et al., 2017c, ApJ, 841, 89

Abbott B. P., et al., 2017d, PhRvD, 96, 022001

Arcavi I., et al., 2017, Natur, 551, 64

Berger E., Fong W., Chornock R., 2013, ApJ, 774, L23

Blackman E. G., Yi I., Field G. B., 1996, ApJ, 473, L79

Blandford R. D., Znajek R. L., 1977, MNRAS, 179, 433

Coulter D. A., et al., 2017, Sci, 358, 1556

Dai Z. G., Gou L. J., 2001, ApJ, 552, 72

D'Avanzo P., et al., 2018, A\&A, 613, L1

Evans P. A., et al., 2017, Sci, 358, 1565

Fernández R., Metzger B. D., 2016, ARNPS, 66, 23

Foucart F., Duez M. D., Kidder L. E., Teukolsky S. A., 2011, PhRvD, 83, 024005

Fraija N., De Colle F., Veres P., Dichiara S., Barniol Duran R., Galvan-Gamez A., 2017, arXiv, arXiv:1710.08514

Goldstein A., et al., 2017, ApJ, 848, L14

Gottlieb O., Nakar E., Piran T., Hotokezaka K., 2018, MNRAS, 479,588

Granot J., Königl A., Piran T., 2006, MNRAS, 370, 1946

Granot J., 2005, ApJ, 631, 1022

Haggard D., Nynka M., Ruan J. J., Kalogera V., Cenko S. B., Evans P., Kennea J. A., 2017, ApJ, 848, L25

Hallinan G., et al., 2017, Sci, 358, 1579

Hinderer T., et al., 2018, arXiv, arXiv:1808.03836

Hu L., et al., 2017, SciBu, 62, 1433

Huang Y. F., Dai Z. G., Lu T., 1999, MNRAS, 309, 513

Ioka K., Toma K., Yamazaki R., Nakamura T., 2006, A\&A, 458, 7

Kasen D., Metzger B., Barnes J., Quataert E., Ramirez-Ruiz E., 2017, Natur, 551, 80

Kasliwal M. M., et al., 2017, Sci, 358, 1559

Kisaka S., Ioka K., Kashiyama K., Nakamura T., 2018, ApJ, 867, 39

Kumar P., Granot J., 2003, ApJ, 591, 1075

Lamb G. P., Kobayashi S., 2017, MNRAS, 472, 4953

Lamb G. P., Kobayashi S., 2018, MNRAS, 478, 733

Lamb G. P., et al., 2019, ApJ, 870, L15

Lazzati D., Perna R., Morsony B. J., Lopez-Camara D., Cantiello 

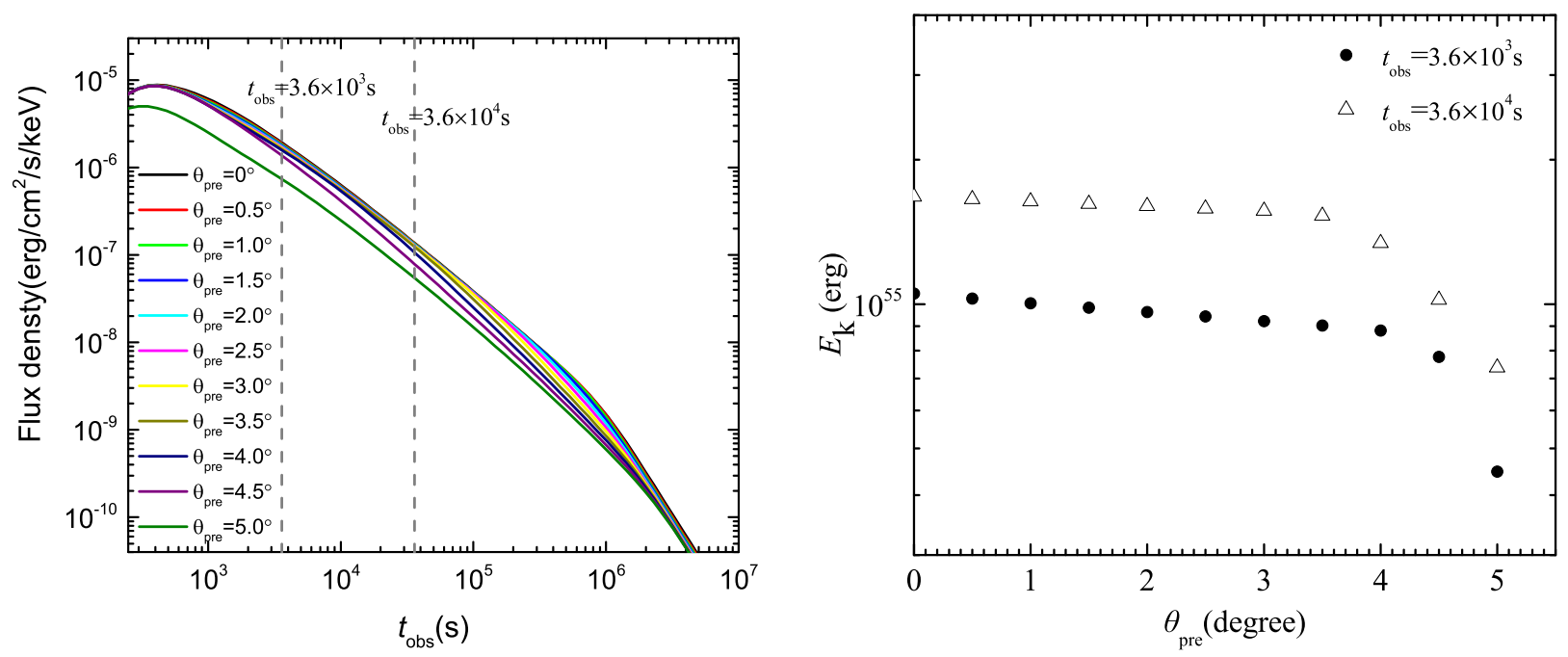

Figure 6. The estimated kinetic energy (right panel) of jets based on X-ray afterglows (left panel) at observed time $t_{\mathrm{obs}}$ for the situations with different $\theta_{\text {pre. }}$.

M., Ciolfi R., Giacomazzo B., Workman J. C., 2018, PhRvL, 120,241103

Lei W. H., Wang D. X., Gong B. P., Huang C. Y., 2007, A\&A, 468,563

Li L.-X., Paczyński B., 1998, ApJ, 507, L59

Lin D.-B., Liu T., Lin J., Wang X.-G., Gu W.-M., Liang E.-W., 2018, ApJ, 856, 90

Lipunov V. M., et al., 2017, ApJ, 850, L1

Liska M., Hesp C., Tchekhovskoy A., Ingram A., van der Klis M., Markoff S., 2018, MNRAS, 474, L81

Liu T., Gu W.-M., Zhang B., 2017, NewAR, 79, 1

Lyman J. D., et al., 2018, NatAs, 2, 751

Margutti R., et al., 2018, ApJ, 856, L18

Meng Y.-Z., et al., 2018, ApJ, 860, 72

Metzger B. D., Berger E., 2012, ApJ, 746, 48

Mooley K. P., et al., 2018, Natur, 554, 207

Murguia-Berthier A., et al., 2017, ApJ, 848, L34

Nousek J. A., et al., 2006, ApJ, 642, 389

Pian E., et al., 2017, Natur, 551, 67

Portegies Zwart S. F., Lee C.-H., Lee H. K., 1999, ApJ, 520, 666

Racusin J. L., et al., 2009, ApJ, 698, 43

Resmi L., et al., 2018, ApJ, 867, 57

Reynoso M. M., Romero G. E., Sampayo O. A., 2006, A\&A, 454, 11

Rossi E., Lazzati D., Rees M. J., 2002, MNRAS, 332, 945

Ruan J. J., Nynka M., Haggard D., Kalogera V., Evans P., 2018, ApJ, 853, L4

Savchenko V., et al., 2016, ApJ, 820, L36

Smartt S. J., et al., 2017, Natur, 551, 75

Soares-Santos M., et al., 2017, ApJ, 848, L16

Song C.-Y., Liu T., Li A., 2018, MNRAS, 477, 21730

Stone N., Loeb A., Berger E., 2013, PhRvD, 87, 084053

Tanvir N. R., et al., 2017, ApJ, 848, L27

Troja E., et al., 2017, Natur, 551, 71

Troja E., et al., 2018, MNRAS, 478, L18

Valenti S., et al., 2017, ApJ, 848, L24

van Eerten E. T. H., et al., 2018, arXiv e-prints, arXiv:1808.06617

Wang X.-G., Zhang B., Liang E.-W., Lu R.-J., Lin D.-B., Li J., Li L., 2018, ApJ, 859, 160

Wu X.-F., Hou S.-J., Lei W.-H., 2013, ApJ, 767, L36

Xiao D., Liu L.-D., Dai Z.-G., Wu X.-F., 2017, ApJ, 850, L41

Xu M., Huang Y. F., 2010, A\&A, 523, A5

Xu M., Huang Y.-F., Kong S.-W., 2008, ChJAA, 8, 411
Yu Y. B., Wu X. F., Huang Y. F., Coward D. M., Stratta G., Gendre B., Howell E. J., 2015, MNRAS, 446, 3642

Zhang B., Mészáros P., 2002, ApJ, 571, 876

Zhang B., Mészáros P., 2004, IJMPA, 19, 2385

Zhang B., Yan H., 2011, ApJ, 726, 90

Zhang B., Fan Y. Z., Dyks J., Kobayashi S., Mészáros P., Burrows D. N., Nousek J. A., Gehrels N., 2006, ApJ, 642, 354

Zhang B., et al., 2007, ApJ, 655, 989

Zhang B.-B., et al., 2018, NatCo, 9, 447

Zou Y.-C., Dai Z.-G., 2006, ChJAA, 6, 551

This paper has been typeset from a $\mathrm{T}_{\mathrm{E}} \mathrm{X} / \mathrm{LAT}_{\mathrm{E}} \mathrm{X}$ file prepared by the author. 